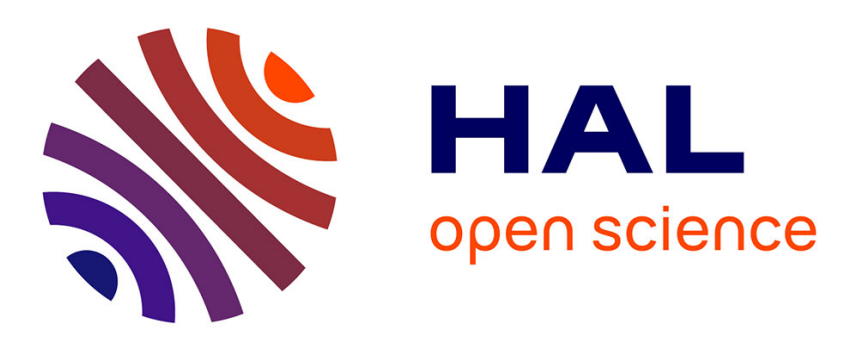

\title{
Etude de l'influence d'un dépôt d'oxyde de cérium sur le comportement vis-à-vis de l'oxydation isotherme et cyclique de deux aciers commerciaux: Z4CN18-09 (AISI 304) et Z2CT17 (F17Ti)
}

\author{
G. Aguilar, J. Larpin, J. Colson
}

\section{To cite this version:}

G. Aguilar, J. Larpin, J. Colson. Etude de l'influence d'un dépôt d'oxyde de cérium sur le comportement vis-à-vis de l'oxydation isotherme et cyclique de deux aciers commerciaux: Z4CN18-09 (AISI 304) et Z2CT17 (F17Ti). Journal de Physique IV Proceedings, 1993, 03 (C9), pp.C9-265-C9-273. 10.1051/jp4:1993925 . jpa-00252363

HAL Id: jpa-00252363 https://hal.science/jpa-00252363

Submitted on 1 Jan 1993

HAL is a multi-disciplinary open access archive for the deposit and dissemination of scientific research documents, whether they are published or not. The documents may come from teaching and research institutions in France or abroad, or from public or private research centers.
L'archive ouverte pluridisciplinaire HAL, est destinée au dépôt et à la diffusion de documents scientifiques de niveau recherche, publiés ou non, émanant des établissements d'enseignement et de recherche français ou étrangers, des laboratoires publics ou privés. 


\title{
Etude de l'influence d'un dépôt d'oxyde de cérium sur le comportement vis-à-vis de l'oxydation isotherme et cyclique de deux aciers commerciaux: Z4CN18-09 (AISI 304) et Z2CT17 (F17Ti)
}

\author{
G. Aguilar, J.P. Larpin et J.C. Colson
}

LRRS Fac. Sci. Mirande, BP 138, F.21004, Dijon Cedex, France

\begin{abstract}
Résumé. - L'addition d'élément actif comme les terres rares est connu pour améliorer le comportement vis-à-vis de l'oxydation à haute température d'aciers chromino-formeurs. Nous présentons ici les résultats relatifs à l'oxydation isotherme à $950^{\circ} \mathrm{C}$ et $1000^{\circ} \mathrm{C}$ et en cyclage thermique à $950^{\circ} \mathrm{C}$ des deux aciers commerciaux Z4CN18-09 (AISI 304) et Z2CT17 (F17Ti) recouverts d'un dépôt fin d'oxyde de cérium réalisé par différentes méthodes "chimiques". Létude du comportement des aciers non traités confirme les résultats de la littérature. Les dépôts de $\mathrm{CeO}_{2}$ ont été réalisés par voie électrophorétique, à partir du sol en suspension dans différents solvants aqueux ou organiques. Ces dépôts, fins et homogènes, ont des effets positifs vis-à-vis de l'oxydation isotherme et cyclique. Il apparait clairement que: 1) en condition isotherme, la vitesse de corrosion diminue légèrement et l'adhérence des couches barrières augmente fortement; 2) en condition de cyclage thermique, l'adhérence de la couche protectrice augmente de façon spectaculaire. La justification de ces résultats réside apparemment dans la formation d'une souscouche de chromine à grains très fins qui confère au système une plasticité plus élevée que précédemment. Ce travail a permis de proposer différents procédés d'introduction superficielle du cérium dans un alliage métallique, de mise en oeuvre beaucoup plus facile et moins couteuse que l'implantation ionique. Les dépôts ainsi obtenus améliorent considérablement l'adhérence des couches protectrices formées sur des aciers inoxydables commerciaux, en particulier lors de leur utilisation dans des installations soumises à des variations de température de grande amplitude.
\end{abstract}

\footnotetext{
Abstract. - Reactive element addition as rare earth elements is a well known method for improving the high temperature oxidation behavior of chromia-former stainless steels. Two commercial stainless steels (AISI 304 and Fel7Ti) were exposed to isothermal oxidation at $950^{\circ} \mathrm{C}$ and $1000^{\circ} \mathrm{C}$ and thermal cycling oxidation at $950^{\circ} \mathrm{C}$ in air under atmospheric pressure. These steels were prepared by coating a thin ceria film by electrophorectic methods or by dipping into a ceria dispersion in various solvants (aqueous or organic). It has been shown for the coated steels that the oxidation rate slightly decreased and that the oxide adherence increased particularly for Fe17Ti steel in isothermal condition. For cyclic conditions, the oxide scale adherence was considerably increased since no scale spallation occured after more than 40 cycles. The observation can be partially explained by the growth of a very fine grained chromia subscale on coated stainless steels. Comparison are made between dipped coating and ion implantation processes for introducing reactive element to the surface. The latter processes provide surface modification which increases the oxide scale adherence of chromiaformer stainless steel, especially when large temperature range variations occur.
} 


\section{Introduction.}

L'addition d'éléments tels que les terres rares, aux aciers est connu pour améliorer leur comportement vis-à-vis de l'oxydation à haute température [1-5]. Cet effet a été mis en évidence sur des alliages implantés le plus souvent avec de l'yttrium [6-11].

Le présent travail est relatif à l'étude de l'effet de dépôts superficiels d'oxydes de cérium réalisés ici par des méthodes chimiques sur deux aciers commerciaux courants. Les résultats comparatifs présentés sont relatifs à l'oxydation isotherme et en cyclage thermique d'alliages Z4CN18-09 et Z2CT17 vierges et revêtus d'un dépôt d'oxyde de cérium.

\section{Caractéristiques des échantillons, méthodes et conditions expérimentales.}

Les alliages étudiés sont des chromino-formeurs que nous connaissons bien. Leurs compositions sont données dans les tableaux I et II.

Tableau I. - Composition pondérale de l'alliage Z4CN18-09 (\% en masse).

\begin{tabular}{|c|c|c|c|c|c|c|c|c|c|c|}
\hline élément & $\mathrm{C}$ & $\mathrm{Mn}$ & $\mathrm{Si}$ & $\mathrm{S}$ & $\mathrm{O}$ & $\mathrm{Cr}$ & $\mathrm{Ni}$ & $\mathrm{Mo}$ & $\mathrm{Cu}$ & $\mathrm{Fe}$ \\
\hline$\%$ & 0,042 & 1,4 & 0,48 & 0,004 & 0,027 & 17,74 & 8,8 & 0,16 & 0,17 & $\mathrm{Bal}$. \\
\hline
\end{tabular}

Tableau II. —Composition pondérale de l'alliage Z2CT17 (F17Ti) (\% en masse).

\begin{tabular}{|c|c|c|c|c|c|c|c|c|c|c|c|}
\hline élément & $\mathrm{C}$ & $\mathrm{Mn}$ & $\mathrm{Si}$ & $\mathrm{S}$ & $\mathrm{P}$ & $\mathrm{Cr}$ & $\mathrm{Ni}$ & $\mathrm{Mo}$ & $\mathrm{Cu}$ & $\mathrm{Ti}$ & $\mathrm{Fe}$ \\
\hline$\%$ & 0,015 & 0,39 & 0,4 & 0,002 & 0,014 & 17,19 & 0,12 & 0,04 & 0,05 & 0,48 & $\mathrm{Bal}$. \\
\hline
\end{tabular}

Les échantillons de $10 \times 10 \times 1 \mathrm{~mm}$ et $23 \times 15 \times 1 \mathrm{~mm}$ ou $0,6 \mathrm{~mm}$ ont été découpés dans la tôle originale puis polis ( 800 ou pâte diamantée de $1 \mu \mathrm{m}$ ).

L'oxydation à haute température $\left(950-1000^{\circ} \mathrm{C}\right)$ a été étudiée en thermobalance sous 1 atmosphère ou $2 \times 10^{4} \mathrm{~Pa}$ d'oxygène pur en régime d'oxydation isotherme ou cyclique $(2 \mathrm{~h}$ de maintien à $950^{\circ} \mathrm{C}, 1 \mathrm{~h}$ à l'ambiante).

Les dépôts d'oxyde de cérium ont été réalisés par diverses méthodes [3-4]:

- Revêtements polymériques réalisés par immersion des échantillons dans une solution colloïdale d'oxyde de cérium, dans une solution aqueuse de polyacrylamide, suivie d'un séchage à $80^{\circ} \mathrm{C}$ et d'une calcination à $800^{\circ} \mathrm{C}$;

- Revêtements alcooliques réalisés par immersion dans une solution alcoolique à $50 \mathrm{~g} / \mathrm{lde}$ nitrate d'ammonium et de cérium;

- Revêtements par électrophorèse en milieu aqueux dans un sol de très fines particules d'hydroxyde de cérium dispersées dans l'eau ou en milieu organique (DMF). Le revêtement obtenu est alors très mince (quelques $\mathrm{nm}$ ).

Tous les échantillons revêtus sont ensuite recuits en montée de température jusqu'à $800^{\circ} \mathrm{C}$ suivi d'un retour lent à l'ambiante. La morphologie de la surface apparaît sur la figure 1. Les profils de concentration des éléments obtenus en utilisant une microsonde ionique (Fig. 2) 
confirment l'incorporation du dépôt d'oxyde de cérium dans l'oxyde "naturel" de l'alliage après recuit. La concentration superficielle en cérium est importante et décroît rapidement. On note également la présence en surface, du fer, un enrichissement en chrome et une accumulation de silicium dans la partie interne du revêtement et dans l'alliage.

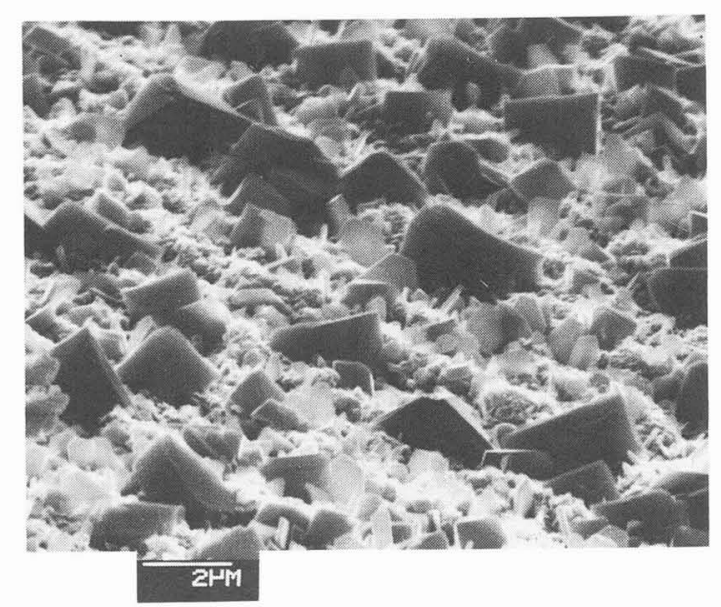

Fig. 1. - Surface de l'acier Z4CN18-09 (AISI 304) revêtu de $\mathrm{CeO}_{2}$ et recuit à $800^{\circ} \mathrm{C}$.

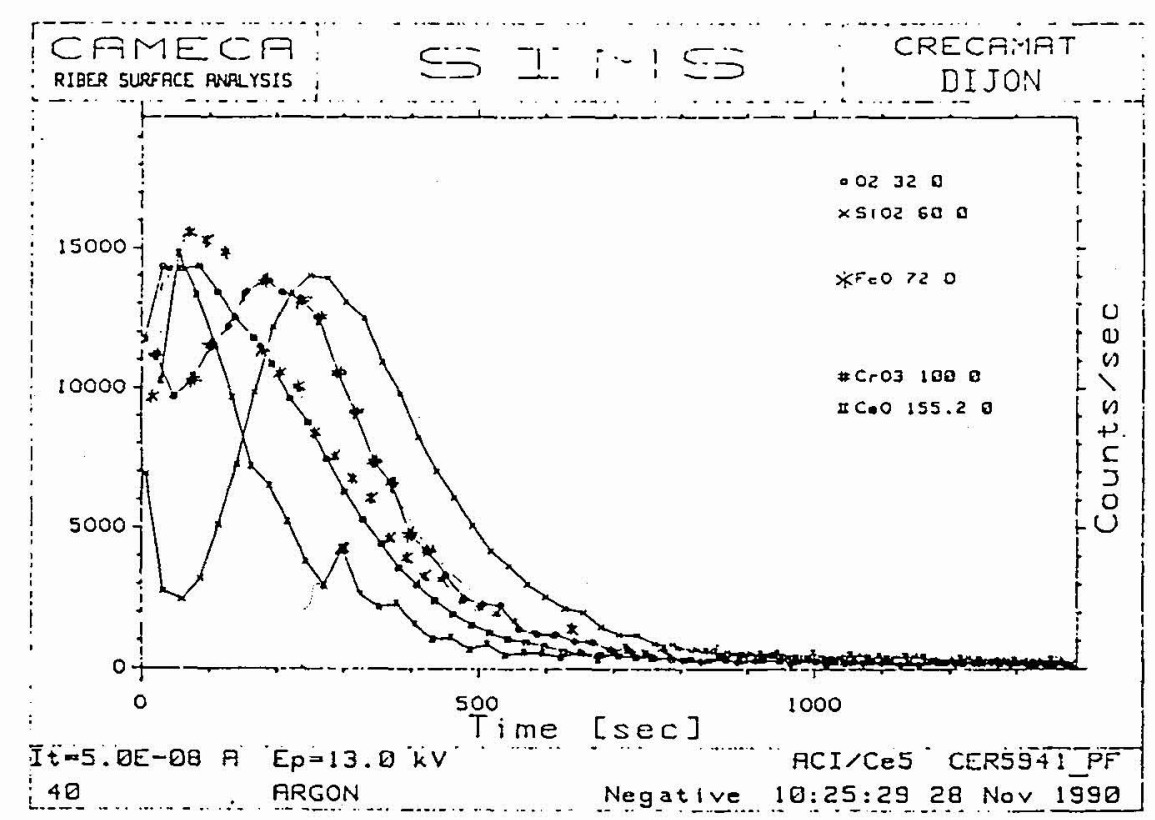

Fig. 2. - Profils de concentration au voisinage de la surface de l'acier Z4CN18-09 (AISI 304) revêtu de $\mathrm{CeO}_{2}$. 


\section{Résultats expérimentaux.}

\subsection{OXYDATION DES ALLIAGES Z4CN18-09 ET Z2CT17 SANS REVÊTEMENTS.}

3.1.1 Cas de l'alliage Z4CN18-09. - Les courbes de marche de l'oxydation à 950 (et $1000{ }^{\circ} \mathrm{C}$ ) présentent une allure parabolique (Fig. 3). Les constantes de vitesse $k p$ déterminées à partir des transformées linéaires $\Delta m / S=f(\sqrt{t})$ sont respectivement égales à $1,8 \times 10^{-12} \mathrm{~g}^{2} \mathrm{~cm}^{-4} \mathrm{~s}^{-1}$ à $950, \mathrm{C}$ et $5,1 \times 10^{-12} \mathrm{~g}^{2} \mathrm{~cm}^{-4} \mathrm{~s}^{-1}$ à $1000^{\circ} \mathrm{C}$ pour un poli 800 .

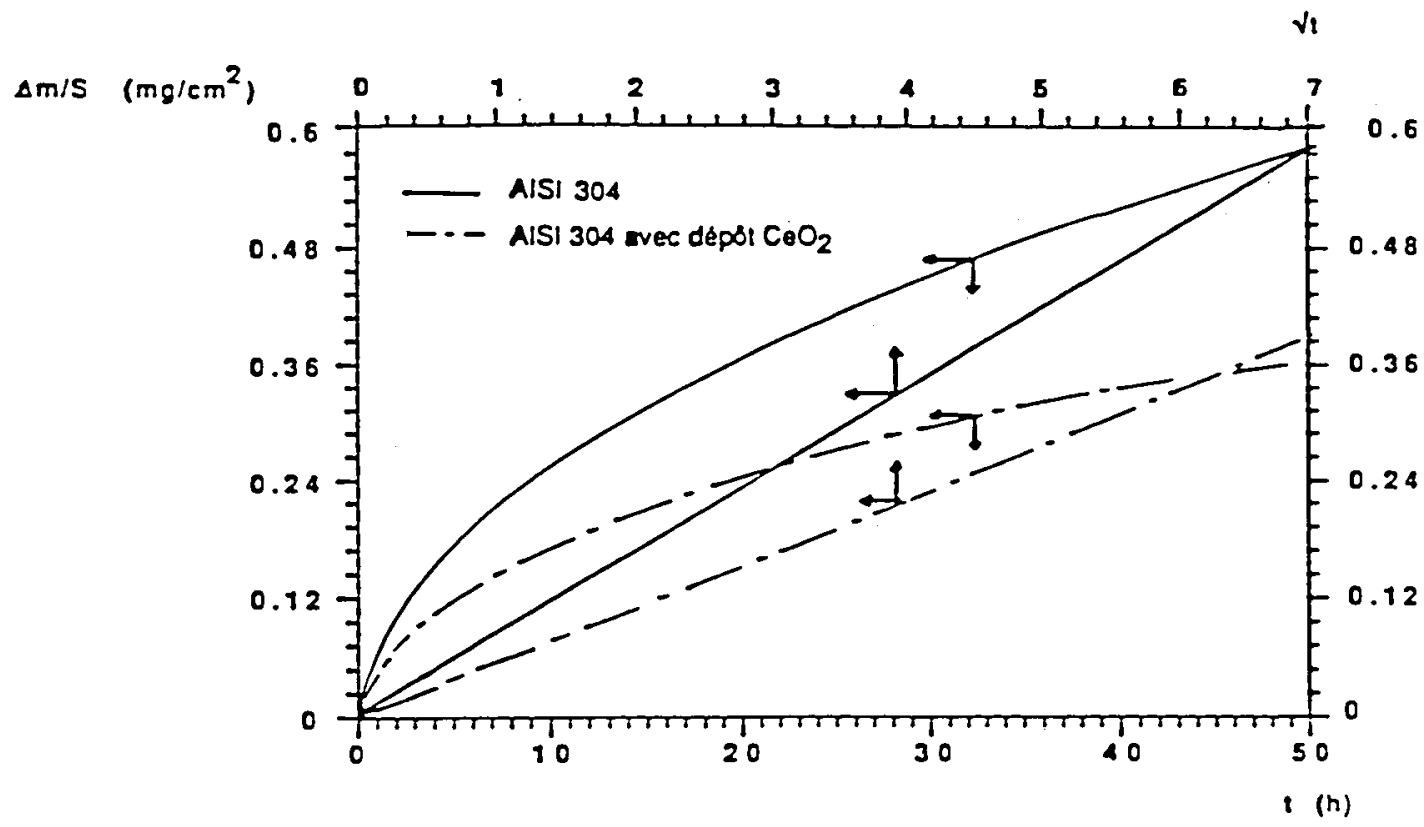

Fig. 3. - Oxydation isotherme de l'acier Z4CN18-09 (AISI 304) revêtu de $\mathrm{CeO}_{2}$ et non revêtu à $950{ }^{\circ} \mathrm{C}$ sous air. Courbes $\Delta m / S=f(t)$ et transformées $\Delta m / S=f(\sqrt{t})$.

On observe initialement une couche de corrosion constituée d'un oxyde de composition proche de $\mathrm{FeCr}_{2} \mathrm{O}_{4}$ puis une sous-couche interne de chromine $\mathrm{Cr}_{2} \mathrm{O}_{3}$ que surmonte une sous-couche constituée de deux phases spinelles proches de $\mathrm{MnFe}_{2} \mathrm{O}_{4}$ et $\mathrm{FeCr}_{2} \mathrm{O}_{4}$.

En régime de cyclage thermique l'allure parabolique des courbes de marche de réaction est conservée (Fig. 4). L'écaillage apparait après 5 ou 7 cycles. La couche barrière, constituée de plusieurs phases spinelles proches de $\mathrm{FeCr}_{2} \mathrm{O}_{4}, \mathrm{MnFe}_{2} \mathrm{O}_{4}$, et de $(\mathrm{Fe}, \mathrm{Cr})_{2} \mathrm{O}_{3}$, perd totalement son caractère protecteur après 25 cycles.

3.1.2 Cas de l'alliage Z2CT17 (F17Ti). - Les courbes de marche de réaction à $950{ }^{\circ} \mathrm{C}$ (et $1000^{\circ} \mathrm{C}$ ) sont également paraboliques (Fig. 5). Quel que soit l'état initial de la surface, la couche d'oxyde formée s'écaille fortement lors du refroidissement. Les coupes transversales de la couche d'oxyde révèlent qu'initialement la couche d'oxyde est constitutée d'oxydes de chrome et de manganèse. Il apparaît ensuite des cristaux de $\mathrm{TiO}_{2}$ dispersés au sein de la couche précédente qui surmonte une sous-couche de chromine à grains très fins $(<0,5 \mu \mathrm{m})$. 


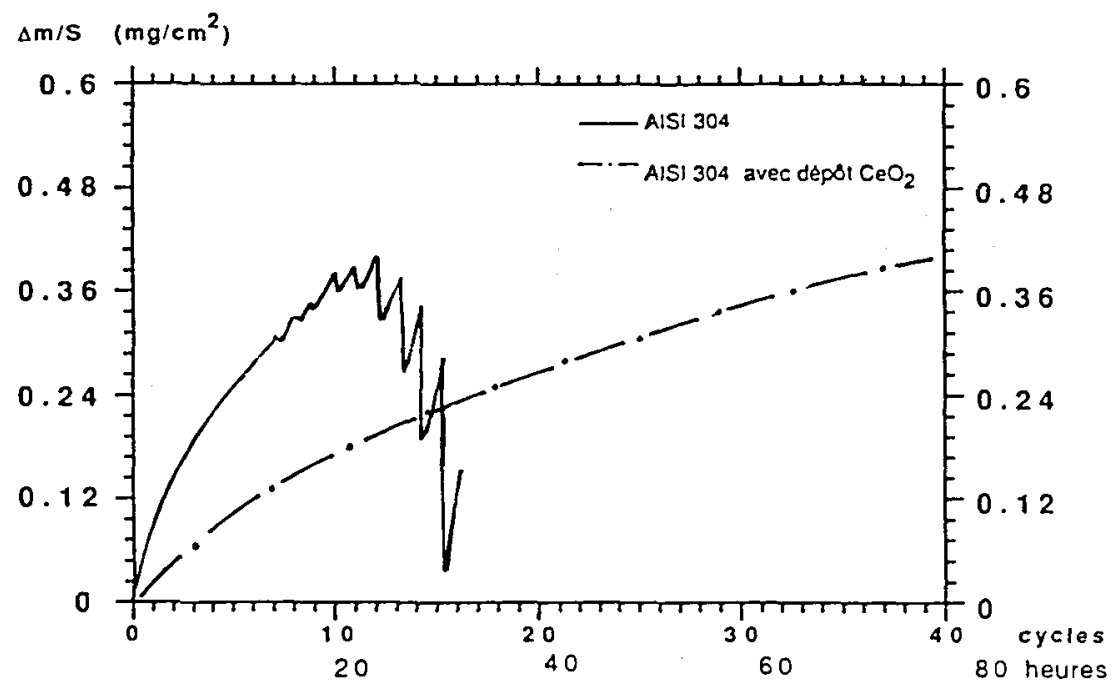

Fig. 4. - Oxydation cyclique de l'acier $\mathrm{Z} 4 \mathrm{CN} 18-09$ (AISI 304) non revêtu et revêtu de $\mathrm{CeO}_{2}$ à $950{ }^{\circ} \mathrm{C}$ sous air.

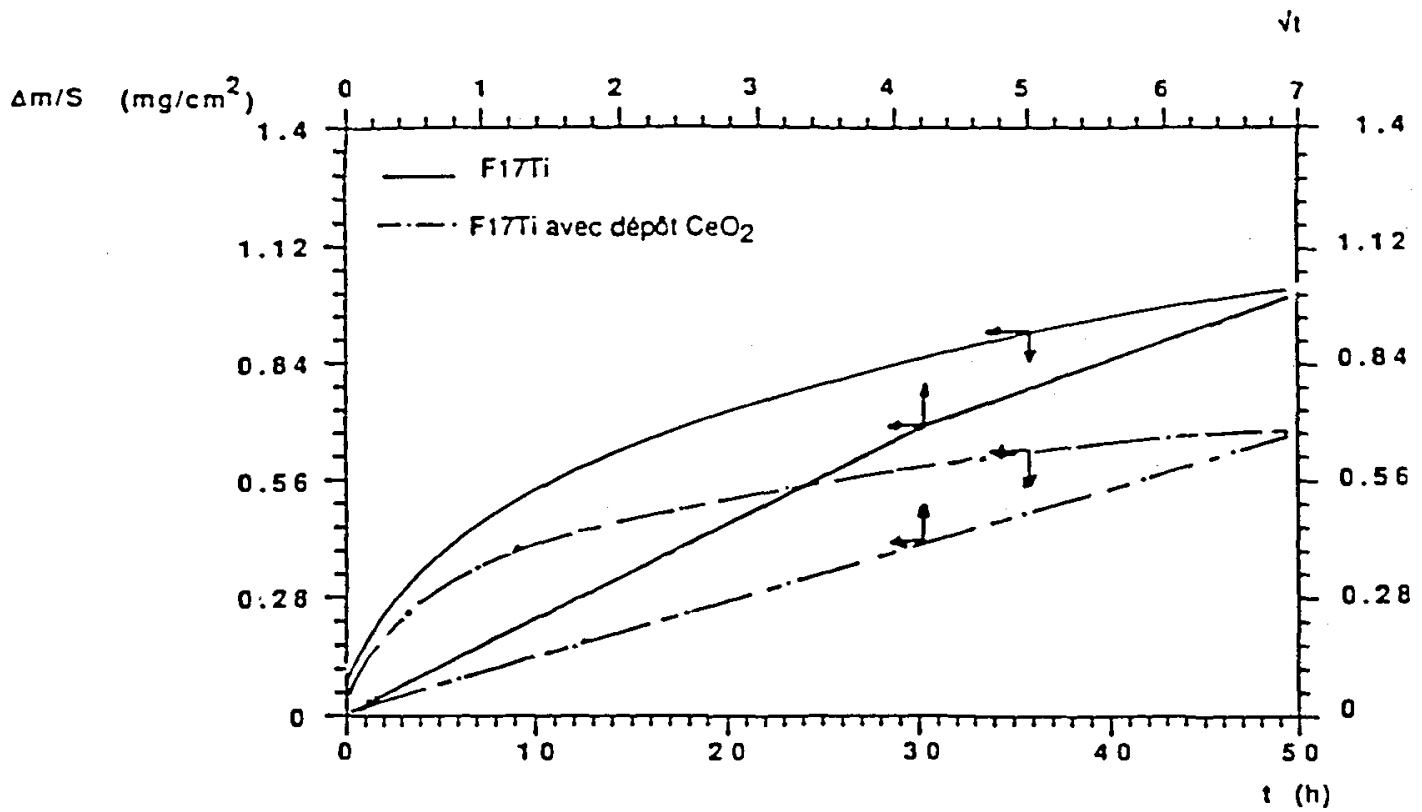

Fig. 5. - Oxydation isotherme de l'acier Z2CT17 (F17TI) revêtu de $\mathrm{CeO}_{2}$ et non revêtu à $950^{\circ} \mathrm{C}$ sous air. Courbes $\Delta m / s=f(t)$ et transformées $\Delta m / S=f(\sqrt{t})$.

Lanalyse par diffraction de rayons $\mathrm{X}$ indique la présence d'une phase proche de $\mathrm{MnFe}_{2} \mathrm{O}_{4}$, de rutile $\mathrm{TiO}_{2}$ et d'une phase proche de $\mathrm{Cr}_{2} \mathrm{O}_{3}$. Il apparaît également une couche enrichie 
en silicium qui pourrait être de la silice amorphe. Les valeurs des constantes de vitesse parabolique sont $0,8 \times 10^{-11} \mathrm{~g}^{2} \mathrm{~cm}^{-4} \mathrm{~s}^{-1}$ à $950^{\circ} \mathrm{C}$ et $1,9 \times 10^{-11} \mathrm{~g}^{2} \mathrm{~cm}^{-4} \mathrm{~s}^{-1}$ à $1000^{\circ} \mathrm{C}$ pour des échantillons poli 800 .

En régime cyclique, un effritement quasi total de la couche barrière apparaît dès le troisième cycle. De ce fait, la vitesse d'oxydation augmente de façon importante (Fig. 6). L'écaillage se produit pour l'essentiel à l'interface interne couche/métal.

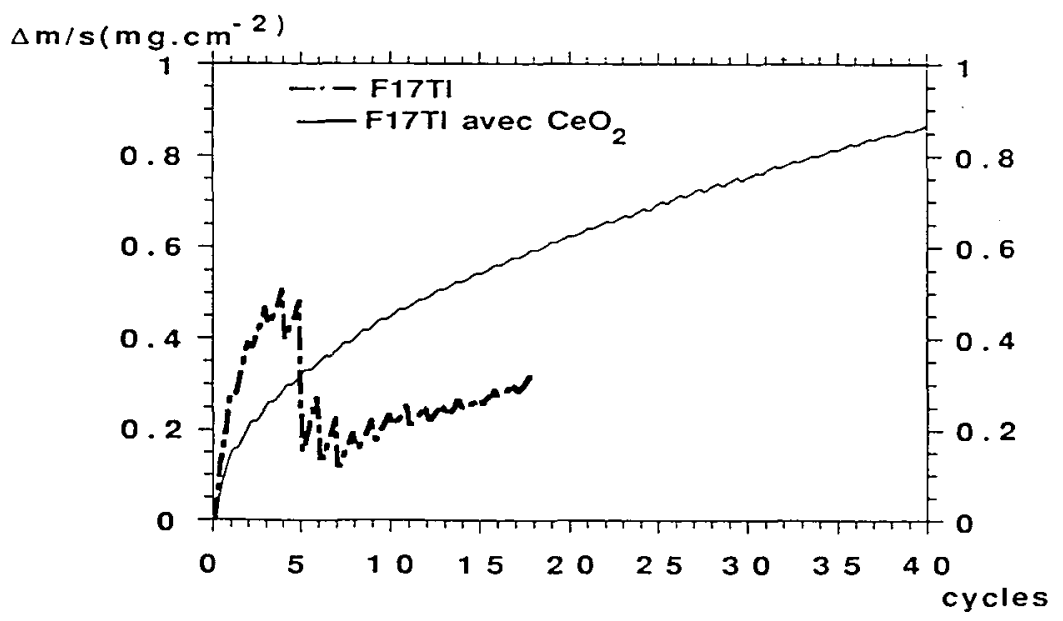

Fig. 6. - Oxydation cyclique de l'acier Z2CT17 (F17TI) non revêtu et revêtu de $\mathrm{CeO}_{2}$ à $950{ }^{\circ} \mathrm{C}$ sous air.

3.2 OXYdATION DES AlLIAGES Z4CN18-09 ET Z2CT17 REVÊTUS DE $\mathrm{CeO}_{2}$. — La qualité des dépôts ayant été optimisée, nous avons finalement retenu de façon privilégiée les dépôts électrophorétiques pour l'ensemble de ce travail.

3.2.1 Cas de l'alliage Z4CN18-09. - Les essais de corrosion réalisés à $950^{\circ} \mathrm{C}$ (et $1000^{\circ} \mathrm{C}$ ) montrent que le régime de corrosion est conservé (Fig. 3). Les constantes de vitesse $k p$ sont abaissées $\left(8,1 \times 10^{-13}\right.$ et $\left.1,8 \times 10^{-12} \mathrm{~g}^{2} \mathrm{~cm}^{-4} \mathrm{~s}^{-1}\right)$. La couche barrière est fortement adhérente au substrat métallique.

La couche d'oxyde obtenue après $50 \mathrm{~h}$ est similaire à celle observée dans le cas de l'alliage non revêtu. On identifie par diffraction $\mathrm{X}$, une phase spinelle de type $\mathrm{MnFe}_{2} \mathrm{O}_{4}$, de la chromine $\mathrm{Cr}_{2} \mathrm{O}_{3}$ et un oxyde complexe contenant du cérium proche de $[(\mathrm{Ca}, \mathrm{Ce})(\mathrm{Ti}, \mathrm{Fe}, \mathrm{Cr}, \mathrm{Mg})]_{21} \mathrm{O}_{38}[12]$.

En régime d'oxydation cyclique (Fig. 4), on assiste à la disparition totale du phénomène d'écaillage même après plus de 60 cycles et la loi d'oxydation reste d'allure parabolique.

3.2.2 Cas de l'alliage Z2CT17. - Les prises de masse des échantillons revêtus sont réduites de près de $50 \%$. Les lois cinétiques identifiées demeurent voisines des précédentes (Fig. 5) mais les échantillons ne présentent aucun écaillage au refroidissement quelle que soit la durée d'oxydation.

Le cérium est identifié au niveau de l'interface entre les deux sous-couches et une ségrégation du titane dans l'alliage est constatée. Lanalyse par diffraction $\mathrm{X}$ de la sur- 
face de la couche d'oxyde formée après $50 \mathrm{~h}$ d'oxydation à $950{ }^{\circ} \mathrm{C}$, révèle la présence d'une phase proche de $\mathrm{MnFe}_{2} \mathrm{O}_{4}$, de $\mathrm{TiO}_{2}$, de $\mathrm{Cr}_{2} \mathrm{O}_{3}$, de $\mathrm{CeO}_{2}$ et d'un oxyde complexe de $[(\mathrm{Ca}, \mathrm{Ce})(\mathrm{Ti}, \mathrm{Fe}, \mathrm{Cr}, \mathrm{Mg})]_{21} \mathrm{O}_{38}[12]$.

Il y a également absence totale d'écaillage en régime cyclique (Fig. 6). L'enveloppe des courbes de marche de réaction demeure parabolique et l'on peut lui attribuer une "constante de vitesse parabolique" dont la valeur moyenne est comparable à celle calculée en condition d'oxydation isotherme.

\section{Discussion.}

Leétude réalisée sur l'alliage $\mathrm{Z} 4 \mathrm{CN} 18-09$ confirme les travaux antérieurs. Les trois produits formés sont $\mathrm{MnFe}_{2} \mathrm{O}_{4}, \mathrm{MnCr}_{2} \mathrm{O}_{4}$ et $\mathrm{Cr}_{2} \mathrm{O}_{3}$; ils constituent des couches denses dont la croissance est réglée par la diffusion cationique, le rôle des joints de grains étant essentiel $[9,11$, 13].

En conditions cycliques les premières manifestations de la relaxation des contraintes thermiques par écaillage apparaissent après 5 à 7 cycles. Elles sont localisées sur une surface relativement faible de l'échantillon (périphérie), puis elles s'étendent à l'ensemble de la surface. Les nouveaux oxydes ont alors des vitesses de croissance élevées.

Le revêtement d'oxyde de cérium diminue la vitesse de corrosion mais ne modifie pas fondamentalement la nature des phases obtenues. On forme une couche de spinelle $(\mathrm{Mn}, \mathrm{Fe}) \mathrm{Cr}_{2} \mathrm{O}_{4}$ emprisonnant l'oxyde de cérium située au-dessus d'une sous-couche de chromine $\mathrm{Cr}_{2} \mathrm{O}_{3}$ à grains extrêmement fins $(70-100 \mathrm{~nm})$. Cette structure de la couche d'oxyde rappelle celle observée lors de l'oxydation de l'alliage 20/25/Nb implanté à l'yttrium par Bennett et al. $[4,5,11]$. L'oxydation de l'alliage semble réglée par la vitesse de croissance de la couche de chromine qui est diminuée de près de $50 \%$. On observe de plus l'apparition d'un oxyde complexe de cérium susceptible de changer le mécanisme réactionnel.

L'absence quasi-totale d'écaillage lors de cyclages thermiques apparaît comme la modification de comportement la plus importante.

Plusieurs explications de ces effets peuvent être avancées telles que l'augmentation de la capacité de déformation plastique de la couche d'oxyde contenant de l'oxyde de cérium et la modification du mécanisme de transport durant la croissance des couches qui constituent la barrière de diffusion $[4,6,11]$. On passerait d'une croissance par diffusion externe des cations à une diffusion interne des anions qui favorise l'adhérence des couches formées.

Lalliage Z2CT17 présente un comportement distinct de celui du Z4CN18-09, les éléments d'addition mineurs modifiant les couches barrières. Il se forme initialement le spinelle $\mathrm{MnCr}_{2} \mathrm{O}_{4}$ emprisonnant dans sa partie superficielle de gros grains de rutile $\mathrm{TiO}_{2}$. L'appauvrissement de la zone interfaciale en manganèse conduit ensuite à la formation de la sous-couche de chromine $\mathrm{Cr}_{2} \mathrm{O}_{3}$. Ces couches toujours couvrantes et protectrices justifient l'allure parabolique des courbes de marche de réaction traduisant des processus diffusionnels cationiques externes. Comme pour l'alliage Z4CN18-09 une ségrégation d'éléments traces dans l'alliage et une dissolution de silicium dans la chromine au niveau de l'interface couche/substrat est détectée. C'est dans cette zone que se produit l'écaillage au choc thermique que les différences entre les coefficients de dilatation de la chromine $\left(5,7 \times 10^{-6} \mathrm{~K}^{-1}\right)$, de la silice $\left(3 \times 10^{-6} \mathrm{~K}^{-1}\right)$ et de l'alliage $\mathrm{Z} 2 \mathrm{CT} 17\left(11 \times 10^{-6} \mathrm{~K}^{-1}\right)$ expliquent en partie.

Le rôle des contraintes induites par une succession de chocs thermiques est parfaitement mis en évidence lors de l'oxydation cyclique. Les échantillons présentent alors des décollements dès les premiers cycles qui provoquent la mise à nu partielle de l'alliage et la formation d'une couche d'oxyde plus riche en $\mathrm{TiO}_{2}$. 
Avec un revêtement d'oxyde de cérium, des modifications de la composition de couches d'oxydes sont observées dès les premières heures de l'oxydation (formation de $\mathrm{Cr}_{2} \mathrm{O}_{3}$ surmontée de spinelle $\mathrm{MnCr}_{2} \mathrm{O}_{4}$, de $\mathrm{TiO}_{2}$ et d'un oxyde complexe de cérium). Le caractère protecteur des couches apparaît clairement. Mais, comme précédemment, des modifications des mécanismes de croissance des couches d'oxydes qui seraient liées à la présence de l'oxyde de cérium dans la couche de spinelle $\mathrm{MnCr}_{2} \mathrm{O}_{4}$ et à la formation de l'oxyde complexe contenant du cérium interviennent. On peut envisager en particulier un ralentissement de la diffusion externe des cations en faveur d'une diffusion interne de l'oxygène, jusqu'à ce que celle-ci devienne prédominante. Cette inversion du mode de transport dans la couche barrière pourrait être à l'origine de l'absence d'écaillage de la couche de corrosion.

Le cyclage thermique ne provoque aucune dégradation spécifique du matériau et les constantes de vitesse d'oxydation cyclique et isotherme demeurent identiques pour un même traitement. Aucun décollement et écaillage n'est constaté après un nombre de cycles important (40 cycles). Il s'agit d'un résultat particulièrement important pour la tenue du matériau à chaud.

\section{Conclusion.}

Létude de l'oxydation des alliages Z4CN18-19 et Z2CT17 à $950^{\circ} \mathrm{C}$ et $1000^{\circ} \mathrm{C}$ nous a permis de constater dans les deux cas la formation de couches de corrosion duplex constituées d'une couche externe de $\mathrm{MnCr}_{2} \mathrm{O}_{4}$ surmontant de la chromine $\mathrm{Cr}_{2} \mathrm{O}_{3}$. Le transfert de matière s'effectue par diffusion externe des cations ce qui conduit à des pertes d'adhérence des couches barrières.

Le rôle important des éléments mineurs $(\mathrm{Mn}, \mathrm{Si}, \mathrm{Ti})$ apparaît clairement en ce qui concerne la nature et les propriétés des couches d'oxyde barrières.

Les dépôts de $\mathrm{CeO}_{2}$ fins et homogènes que nous avons réalisés sur ces alliages par des procédés chimiques simples ont tous des effets très positifs sur la vitesse de corrosion oxydante isotherme ou cyclique. Ils diminuent de façon significative la vitesse de corrosion et augmentent l'adhérence des couches barrières en condition isotherme et surtout en régime de cyclage thermique puisque aucun écaillage n'est constaté après 5 jours de tests.

Ces résultats semblent pouvoir s'expliquer par la modification du mode de transport de matière lors de la croissance de la couche de corrosion qui serait due a un changement de nature des défauts ponctuels dominants dans l'oxyde protecteur. Il en résulterait une augmentation de l'adhérence de ces couches puisqu'il n'y aurait plus d'accumulation de porosités localisées à l'interface interne. Bennett et al. $[5,7,11]$ ont fait des observations voisines qui les conduisent à des conclusions similaires pour un alliage du même type enrichi en terres rares par implantation.

Par contre, nous n'avons pas observé de ségrégations du cérium aux joints de grains de la chromine pouvant bloquer la diffusion des cations, comme l'envisagent certains auteurs $[4,7-10]$.

En l'état actuel, les raisons profondes du changement de mécanisme de transport de matière proposé, restent à établir. L'identification d'un oxyde complexe contenant du cérium constitue une piste qu'il convient maintenant d'approfondir.

Le présent travail [14] permet donc de proposer des revêtements anticorrosion dont la mise en oeuvre par des procédés chimiques et électrochimiques est à la fois simple et peu coûteuse. Ces dépôts constitués d'oxyde de cérium augmentent considérablement l'adhérence des couches protectrices formées sur des aciers inoxydables commerciaux et ralentissent leur vitesse de dégradation à haute température en régime isotherme et surtout en régime de 
cyclage thermique.

Il s'agit certainement de procédés qui connaitront des développements appliqués à court terme dans différents domaines de la corrosion par les gaz à haute température.

\section{Remerciements.}

Ce travail a été réalisé en collaboration avec le Laboratoire d'Electrochimie de l'Université de Franche Comté URA CNRS 436.

\section{Bibliographie}

[1] Allan I.M., WhitTle D.P., Stringer J., Oxid. Met. 12 (1978) 35.

[2] Delaunay D., Huntz A.M., Lacombe P., Corros. Sci. 20 (1980) 1109.

[3] RHYS-JONES T.N., GRABKE H.J., Mater. Sci. Technol. 4/5 (1988) 446.

[4] Bennett M.J., Bellamy B.A., Knights C.F., Meadows N., Eyre N.J., Mater. Sci. Eng. 69 (1985) 359.

[5] MOON D.P., BENNETT M.J., Mater. Sci. Forum 43 (1989) 269.

[6] Cotell C.M., Yurek G.J., Hussey R.J., Mitchell D.F., Graham M.J., J. Electrochem. Soc. 134 (1987) 1871.

[7] MoON D.P., Oxid. Met. 32 (1/2) (1989) 47.

[8] Mrowec S., Jedlinski J., GIL A., Mater. Sci. Eng. A120 (1989) 169.

[9] Cotell C.M., Yurek G.J., Hussey R.J., Mitchell D.F., Graham M.J., Oxid. Met. 34 (3/4) (1990) 173.

[10] Przybylski K., Garratt-Reed A.J., Yurek G.J., J. Electrochem. Soc. 135 (1988) 508.

[11] BENNETT M.J., MOON D.P., The role of active elements in the oxidation behaviour of high temperature metals and alloys, E. Lang ed. (Elsevier Appl. Science, Londres et New York, 1988) p. 111.

[12] GateHouse B., Am. Mineral. 63 (1978) 28.

[13] Mitchell D.F., Hussey R.J., Graham M.J.,J. Vac. Sci. Technol. A 2 (1984) 789.

[14] Aguilar G., Thèse Dijon, Université de Bourgogne (octobre 1991). 